\begin{tabular}{|c|c|c|}
\hline & $\begin{array}{c}\text { Gazi University } \\
\text { Journal of Science }\end{array}$ & 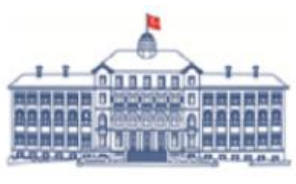 \\
\hline & http://dergipark.gov.tr/gujs & \\
\hline
\end{tabular}

\title{
Best Proximity Point Results for Multivalued Cyclic Mappings on Partial Metric Spaces
}

\author{
Mustafa ASLANTAS* \\ Çankırı Karatekin University, Department of Mathematics, 18100, Çankır, Turkey
}

\section{Highlights}

- This paper focuses on cyclic contraction mapping.

- Some properties of this mapping are examined.

- New best proximity point results were obtained for this mapping.

\begin{tabular}{l} 
Article Info \\
\hline $\begin{array}{l}\text { Received: } 24 \text { Oct } 2020 \\
\text { Accepted:16 June } 2021\end{array}$ \\
Keywords \\
\hline $\begin{array}{l}\text { Best proximity point } \\
\text { Multivalued mapping } \\
\text { Partial metric space }\end{array}$
\end{tabular}

\begin{abstract}
Let $\emptyset \neq \hat{\mathrm{R}}$, Ś be subsets of a partial metric space $(\Omega, \vartheta)$ and $\Psi: \mathrm{R} \rightarrow$ Ś be a mapping. If $\mathrm{R} \cap S=\emptyset$, it cannot have a solution of equation $\Psi_{\varsigma}=\varsigma$ for some $\varsigma \in \hat{R}$. Hence, it is sensible to investigate if there is a point $\ddot{\eta}$ satisfying $\vartheta(\dddot{\eta}, \Psi \dddot{\eta})=\vartheta(\hat{R}, S)$ which is called a best proximity point. In this paper, we first introduce a concept of Hausdorff cyclic mapping pair. Then, we revise the definition of 0-boundedly compact on partial metric spaces. After that, we give some best proximity point results for these mappings. Hene, our results combine, generalize and extend many fixed point and best proximity point theorems in the literature as properly. Moreover, a comparative and illustrative example to demonstrate the effectiveness of our results has been presented.
\end{abstract}

\section{INTRODUCTION}

In 1922, Banach [1] obtained a very significant result, known as the Banach contraction principle, on complete metric spaces. Because of its applicable in various fields of nonlinear analysis and applied, this the result has been extended in different ways [2-6]. Nadler [7] proved one of the famous and interesting generalizations by considering multivalued mappings.

Theorem 1. Let $\Psi: \Omega \rightarrow C B(\Omega)$ be a multivalued mapping on a complete metric space $(\Omega, \sigma)$. If there is $q$ in $[0,1)$ satisfying

$H_{\sigma}(\Psi \ddot{\eta}, \Psi \ddot{u}) \leq q \sigma(\dddot{\eta}, \ddot{u})$

for all $\ddot{\eta}, \ddot{v} \in \Omega$ where

$C B(\Omega)=\{R \in \subseteq: R$ is closed and bounded in $(\Omega, \sigma)\}$

and $H$ is a Hausdorff metric w.r.t. $\sigma$ on $C B(\Omega)$, then is a point $\dddot{\eta}^{*}$ in $\Omega$ satisfying $\dddot{\eta}^{*}=\Psi \dddot{\eta}^{*}$.

Introducing the nice definition of cyclic mapping, Kirk et al. [8] proved another generalization of Banach's principle.

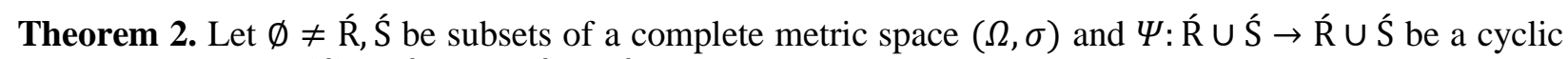
mapping, that is, $\Psi(\hat{\mathrm{R}}) \subseteq S$ and $\Psi(S ́) \subseteq \mathrm{R}$. If there is q in $[0,1)$ satisfying 
$\sigma(\Psi \ddot{\eta}, \Psi \ddot{u}) \leq q \sigma(\ddot{\eta}, \mathrm{u})$

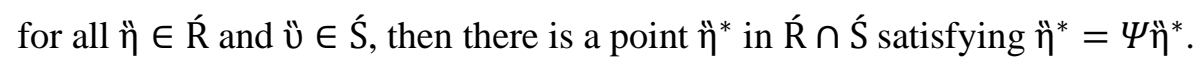

Note that, unlike the mentioned principle, $\Psi$ may not be continuous in Theorem 2 . Hence, the continuity condition of $\Psi$ has been neglected. Therefore, this topic is generalized and extended in different ways $[9,10]$. Also, considering a similar approach the following result has been obtained.

Theorem 3. [8] Let $\emptyset \neq$ R, Ś be subsets of a complete metric space $(\Omega, \sigma)$ where Ŕ, Ś are closed. Assume that $\Psi: \hat{\mathrm{R}} \rightarrow S^{\mathrm{S}}$ and $\Phi: S \in R$ are two mappings such that

$\sigma(\Psi \dddot{\eta}, \Phi \ddot{)}) \leq q \sigma(\ddot{\eta}, \mathrm{v})$

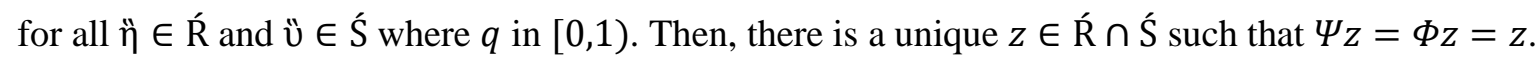

Recently, some other extensions of Banach's result have been obtained by considering nonself mappings. Let $\emptyset \neq \hat{\mathrm{R}}$, Ś be subsets of a metric space $(\Omega, \sigma)$ and $\Psi: \hat{\mathrm{R}} \rightarrow S^{\mathrm{S}}$ be a mapping. If $\hat{\mathrm{R}} \cap \hat{\mathrm{S}}=\varnothing$, then $\Psi$ cannot have a fixed point. Hence, because of the fact that $\sigma(\grave{\eta}, \Psi \dddot{\eta}) \geq \sigma(\hat{R}, S)$ for all $\ddot{\eta} \in R$, it is logical to search a point $\ddot{\eta}$ satisfying $\sigma(\ddot{\eta}, \Psi \dddot{\eta})=\sigma(\hat{\mathrm{R}}, \hat{S})$ which is called a best proximity point [11]. Since every best proximity point is a natural generalization of fixed point in case of $\hat{R}=S=\Omega$, many authors have studied on this topic in literature [12-20].

Taking into account $R \dot{R} \cap S^{=} \emptyset$ in the Theorem 2, Eldered and Veeramani [21] gave the nice concept, so called cyclic contraction mapping, and proved some best proximity point results for such mappings.

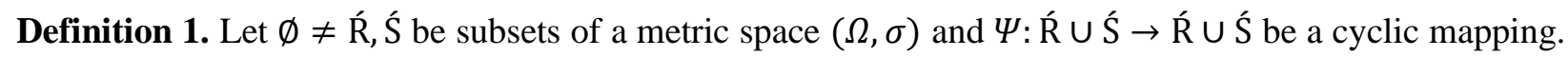
If there is $q$ in $[0,1)$ satisfying

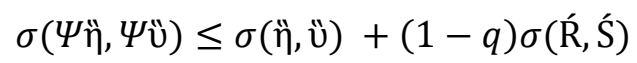

for all $\dddot{\eta} \in R$ and $u ̈ \in S$, then $\Psi$ is called cyclic contraction mapping.

Theorem 4. [21] Let $\emptyset \neq R$ R, Ś be subsets of a metric space $(\Omega, \sigma)$ and where Ŕ, Ś are closed. Assume that

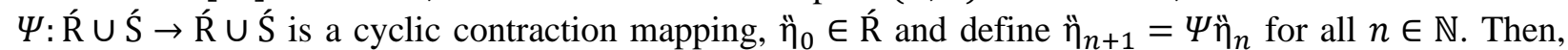
there is $\ddot{\eta} \in$ Ŕ satisfying $\sigma(\ddot{\eta}, \Psi \dddot{\eta})=\sigma(\hat{\mathrm{R}}, \hat{S})$ provided that $\left\{\ddot{\eta ̆}_{2 n-1}\right\}$ has a convergent subsequence in $\mathrm{R}$.

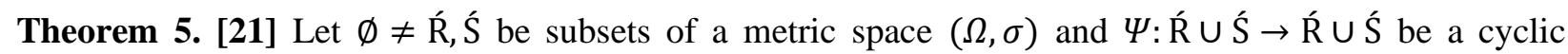

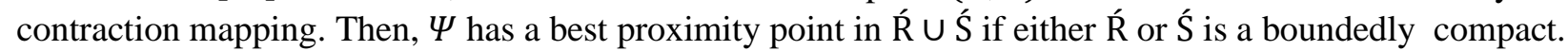

On the other hand, Matthews [22] introduced the definition of partial metric:

Definition 2. Let $\Omega \neq \emptyset$ and $\vartheta: \Omega \times \Omega \rightarrow[0, \infty)$ be a function. If the following conditions hold:

p1) $\vartheta(\ddot{\eta}, \ddot{\eta})=\vartheta(\ddot{\eta}, \ddot{u})=\vartheta(\ddot{v}, \ddot{v})$ if and only if $\ddot{\eta}=\ddot{v}$,

p2) $\vartheta(\ddot{\eta}, \ddot{\eta}) \leq \vartheta(\ddot{\eta}, \ddot{u})$,

p3) $\vartheta(\ddot{\eta}, \ddot{u})=\vartheta(\ddot{u}, \ddot{\eta})$,

p4) $\vartheta(\ddot{\eta}, z) \leq \vartheta(\ddot{\eta}, \ddot{v})+\vartheta(\ddot{v}, z)-\vartheta(\ddot{v}, \ddot{v})$,

for all $\ddot{\eta}, \ddot{v}, z \in \Omega$, then $\vartheta$ is called a partial metric. Also, the pair $(\Omega, \vartheta)$ is said to be partial metric space.

For simplicity, in the rest of this paper, we will use pms instead of partial metric space. It can be seen that every metric space is a pms, but every pms may not be metric space. In fact, let $\Omega=[0, \infty)$ and a function 
$\vartheta: \Omega \times \Omega \rightarrow[0, \infty)$ defined by $\vartheta(\ddot{\eta}, \ddot{u})=\max \{\ddot{\eta}, \ddot{u}\}$ for all $\ddot{\eta}, \imath \ddot{v} \in \Omega$. Then, $\vartheta$ is a partial metric, but it is not a metric. For other example of pms, we refer to [23-25].

Let $(\Omega, \vartheta)$ be a pms. Then, $\vartheta$ generates $T_{0}-\operatorname{topology} \tau_{\vartheta}$ on $\Omega$ which has as a base the family open $\vartheta$-balls $\left\{B_{\vartheta}(\ddot{\eta}, \varepsilon): \ddot{\eta} \in \Omega, \varepsilon>0\right\}$

where

$B_{\vartheta}(\ddot{\eta}, \varepsilon)=\{\ddot{v} \in \Omega: \vartheta(\ddot{\eta}, \ddot{v})<\vartheta(\ddot{\eta}, \ddot{\eta})+\varepsilon\}$

for all $\ddot{\eta} \in \Omega$ and $\varepsilon>0$.

Let $\left\{\ddot{\eta}_{n}\right\}$ be a sequence in $\Omega$ and $\ddot{\eta} \in \Omega$. It is clear that $\left\{\ddot{\eta}_{n}\right\}$ converges to $\ddot{\eta}$ w.r.t. $\tau_{\vartheta}$ if and only if $\lim _{n \rightarrow \infty} \vartheta\left(\ddot{\eta}_{n}, \ddot{\eta}\right)=\vartheta(\ddot{\eta}, \ddot{\eta})$

If $\lim _{n, m \rightarrow \infty} \vartheta\left(\ddot{\eta}_{n}, \ddot{\eta}_{m}\right)$ is finite and exists, then $\left\{\ddot{\eta}_{n}\right\}$ is called a Cauchy sequence. Then $(\Omega, \vartheta)$ is called a complete pms if every Cauchy sequence $\left\{\ddot{\eta}_{n}\right\}$ in $\Omega$ converges to a point $\grave{\eta}$ in $\Omega$ satisfying

$\lim _{n, m \rightarrow \infty} \vartheta\left(\ddot{\eta}_{n}, \dddot{\eta}_{m}\right)=\vartheta(\ddot{\eta}, \grave{\eta})$

Recently, Romaguera [26] introduced the concept of 0-complete pms. Hence, a weaker form of completeness on pms has been obtained.

Definition 3. Let $\left\{\ddot{\eta}_{n}\right\}$ be a sequence in a pms $(\Omega, \vartheta)$.

i) If $\lim _{n, m \rightarrow \infty} \vartheta\left(\ddot{\eta}_{n}, \ddot{\eta}_{m}\right)=0$, then $\left\{\ddot{\eta}_{n}\right\}$ is called 0-Cauchy sequence

ii) If every 0 -Cauchy sequence converges to a point $\ddot{\eta}$ in $\Omega$ w.r.t. $\tau_{\vartheta}$ such that

$\lim _{n, m \rightarrow \infty} \vartheta\left(\dddot{\eta}_{n}, \dddot{\eta}_{m}\right)=\vartheta(\dddot{\eta}, \dddot{\eta})=0$,

then $(\Omega, \vartheta)$ is called 0 -complete pms.

Because of the fact that every 0 -Cauchy sequence in $\Omega$ is a Cauchy sequence, every complete pms is 0 complete. But, the converse may not be true. Indeed, let us consider the set $(\Omega=\mathbb{Q} \cap[0, \infty), \vartheta)$ is a pms where $\vartheta(\ddot{\eta}, \ddot{v})=\max \{\ddot{\eta}, \mathfrak{u}\}$ for all $\ddot{\eta}, \ddot{v} \in \Omega$. Then, $(\Omega, \vartheta)$ is 0 -complete but it is not a complete pms.

Definition 4. [27] Let $(\Omega, p)$ be a pms and $\emptyset \neq \hat{\mathrm{R}} \subseteq \Omega$. Ŕ is called a 0 -boundedly compact if every bounded sequence $\left\{\ddot{\eta}_{n}\right\}$ has a subsequence $\left\{\ddot{\eta}_{n_{k}}\right\}$ such that

$$
\lim _{n \rightarrow \infty} \vartheta\left(\ddot{\eta}_{n_{k}}, \grave{\eta}\right)=\vartheta(\grave{\eta}, \grave{\eta})=0 \text {. }
$$

If $\vartheta$ is a partial metric on $\Omega$, then the mapping $\vartheta^{s}: \Omega \times \Omega \rightarrow[0, \infty)$ defined by

$\vartheta^{s}(\grave{\eta}, \grave{u})=2 \vartheta(\dddot{\eta}, \ddot{u})-\vartheta(\dddot{\eta}, \grave{\eta})-\vartheta(\ddot{v}, \ddot{u})$

for all $\ddot{\eta}, \ddot{\cup} \in \Omega$ is an ordinary metric on $\Omega$.

Now, we give the relations between pms $(\Omega, \vartheta)$ and corresponding metric space $\left(\Omega, \vartheta^{S}\right)$..

Lemma 1. [28] Let $\left\{\ddot{\eta}_{n}\right\}$ be a sequence in a pms $(\Omega, \vartheta)$. 
i) $\left\{\ddot{\eta}_{n}\right\}$ is a Cauchy sequence in $\left(\Omega, \vartheta^{s}\right)$ iff $\left\{\ddot{\eta}_{n}\right\}$ is a Cauchy sequence in $(\Omega, \vartheta)$.

ii) $\left(\Omega, \vartheta^{S}\right)$ is a complete metric space iff $(\Omega, \vartheta)$ is a complete pms.

iii) Given a sequence $\left\{\ddot{\eta}_{n}\right\}$ in $\Omega$ and $\ddot{\eta} \in \Omega$. Then, we get

$\lim _{n \rightarrow \infty} \vartheta^{s}\left(\ddot{\eta}_{n}, \ddot{\eta}\right)=0 \Leftrightarrow \vartheta(\ddot{\eta}, \ddot{\eta})=\lim _{n \rightarrow \infty} \vartheta\left(\ddot{\eta}_{n}, \ddot{\eta}\right)=\lim _{n, m \rightarrow \infty} \vartheta\left(\ddot{\eta}_{n}, \ddot{\eta}_{m}\right)$

Using Lemma 1 (iii), it is clear that

$\lim _{n \rightarrow \infty} \vartheta^{S}\left(\ddot{\eta}_{n}, \grave{\eta}\right)=0=\lim _{n \rightarrow \infty} \vartheta^{S}\left(\ddot{v}_{n}, \ddot{v}\right) \Leftrightarrow \lim _{n \rightarrow \infty} \vartheta\left(\ddot{\eta}_{n}, \ddot{v}_{n}\right)=\vartheta(\grave{\eta}, \grave{u})$

Now, we give the following lemmas which are very useful in our main results.

Lemma 2. [28] Let $(\Omega, \vartheta)$ be a pms and $\mathrm{R}, \hat{S} \in C B^{\vartheta}(\Omega)$. Then, for each $a \in R$ and $\varepsilon>0$, there exists $b \in$ Ś such that

$\vartheta(a, b) \leq H_{\vartheta}(\hat{R}, S ́)+\varepsilon$.

Lemma 3. [28] Let $\emptyset \neq \hat{R}$ be a subset of a pms $(\Omega, \vartheta)$. Then, we have

$\ddot{\eta} \in \bar{R} \Leftrightarrow \vartheta(\ddot{\eta}, \hat{R})=\vartheta(\ddot{\eta}, \ddot{\eta})$

where $\bar{R}$ is closure of Ŕ w.r.t. $\tau_{\vartheta}$.

Let $C B^{\vartheta}(\Omega)=\{\hat{\mathrm{R}} \subseteq \Omega$ : Ŕ is closed and bounded in $(\Omega, \vartheta)\}$.

Aydi et al. [28] defined partial Hausdorff metric of $(\Omega, \vartheta)$ on $C B^{\vartheta}(\Omega)$. The function

$H_{\vartheta}: C B^{\vartheta}(\Omega) \times C B^{\vartheta}(\Omega) \rightarrow[0, \infty)$

defined by

$H_{\vartheta}(\hat{\mathrm{R}}, \hat{S})=\max \left\{\sup _{a \in \mathrm{R}} \vartheta(a, \dot{S}), \sup _{b \in \hat{S}} \vartheta(\hat{\mathrm{R}}, b)\right\}$

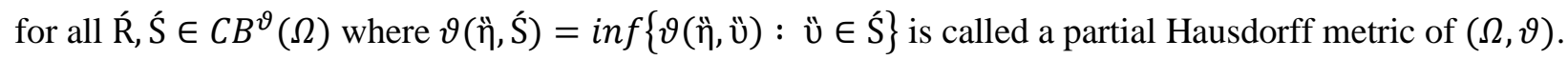

The properties of partial Hausdorff metric were given in [28]:

Lemma 4. [28] Let $(\Omega, \vartheta)$ be a pms. For all Ŕ, Ś $\in C B^{\vartheta}(\Omega)$, we have

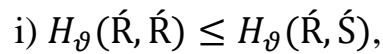

ii) $H_{\vartheta}(\hat{R}, S ́)=H_{\vartheta}(\hat{S}, \hat{R})$,

iii) $H_{\vartheta}(\hat{\mathrm{R}}, \hat{S}) \leq H_{\vartheta}(\hat{\mathrm{R}}, C)+H_{\vartheta}(C, \mathrm{~S})-\inf f_{c \in C} \vartheta(c, c)$,

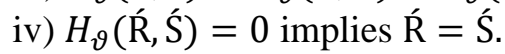

Then, they obtained the following result.

Theorem 6. [28] Let $\Psi: \Omega \rightarrow C B^{\vartheta}(\Omega)$ is a multivalued mapping on a complete pms $(\Omega, \vartheta)$. If there is $q$ in $[0,1)$ satisfying

$H_{\sigma}(\Psi \ddot{\eta}, \Psi \ddot{v}) \leq q \vartheta(\ddot{\eta}, \ddot{v})$ 
for all $\dddot{\eta}, \grave{v} \in \Omega$, then there is a point $\dddot{\eta}^{*}$ in $\Omega$ satisfying $\dddot{\eta}^{*}=\Psi \dddot{\eta}^{*}$.

For any subset $\mathrm{R}$ of $\Omega$, image of Ŕ under the multivalued mapping $\Psi$ is defined as

$\Psi(\hat{\mathrm{R}})=\bigcup_{\grave{\eta} \in \hat{\mathrm{R}}} \Psi \ddot{\eta}$.

Now, we remember the basic concepts and notations of best proximity point theory.

Let $(\Omega, \vartheta)$ be a pms, Ŕ, Ś be nonempty subsets of $\Omega$ and $\Psi: R \rightarrow S ́$ be a mapping. We regard the following subsets of Ŕ and Ś, respectively:

$\dot{\mathrm{R}}_{0}=\{\ddot{\eta} \in \mathrm{R}: \vartheta(\ddot{\eta}, \mathrm{ü})=\vartheta(\hat{\mathrm{R}}, \hat{S})$ for some $\mathrm{u} \in \mathrm{S}\}$

and

$S_{0}=\{\ddot{u} \in S: \vartheta(\dddot{\eta}, u ̈)=\vartheta(R ́, S ́)$ for some $\ddot{\eta} \in R\}$

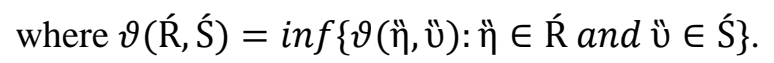

In this paper, we first introduce a concept of Hausdorff cyclic mapping pair. Then, we revise the definition of 0-boundedly compact on partial metric spaces. After that, we give some best proximity point results for these mappings. Hene, our results combine, generalize and extend many fixed point and best proximity point theorems in the literature as properly. Moreover, a comparative and illustrative example to demonstrate the effectiveness of our results has been presented.

\section{MAIN RESULTS}

In this section, we introduce Hausdorff cyclic mapping pair for multivalued mappings.

Definition 5. Let $\emptyset \neq R$, Ś be subsets of a pms $(\Omega, \vartheta)$. Assume that $\Psi: R \rightarrow C B^{\vartheta}(\hat{\mathrm{S}})$ and $\Phi: S \rightarrow C B^{\vartheta}(\hat{\mathrm{R}})$ are multivalued mappings. If there exists $q$ in $(0,1)$ such that

$H_{\boldsymbol{\sigma}}(\Psi \ddot{\eta}, \Phi u ̈) \leq q \vartheta(\ddot{\eta}, \ddot{u})+(1-q) \vartheta(\hat{\mathrm{R}}, \dot{S})$

for all $\dddot{\eta} \in R$ and $u \in \in$ Ś, then the pair $(\Psi, \Phi)$ is called Hausdorff cyclic contraction mapping pair.

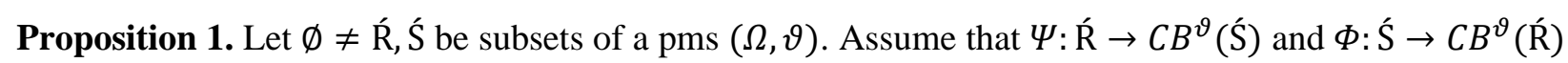
are multivalued mappings satisfying the pair $(\Psi, \Phi)$ is a Hausdorff cyclic contraction mapping pair and $\ddot{\eta}_{0} \in R$ R. Consider the sequence $\left\{\ddot{\eta}_{n}\right\}$ in Ŕ $\cup$ Ś by $\ddot{\eta}_{2 n+1} \in \Psi \ddot{\eta}_{2 n}$ and $\ddot{\eta}_{2 n+2} \in \Phi \ddot{\eta}_{2 n+1}$ for all $n \in \mathbb{N}$. Then, we have $\vartheta\left(\dddot{\eta}_{n}, \ddot{\eta}_{n+1}\right) \rightarrow \vartheta(\hat{R}, S ́)$ as $n \rightarrow \infty$.

Proof. Choose $\dddot{\eta}_{1} \in \Psi \ddot{\eta}_{0}$. From (1), there exists $q$ in $(0,1)$ such that

$H_{\vartheta}\left(\Psi \ddot{\eta}_{0}, \Phi \ddot{\eta}_{1}\right) \leq q \vartheta\left(\ddot{\eta}_{0}, \ddot{\eta ̆}_{1}\right)+(1-q) \vartheta(\hat{\mathrm{R}}, \mathbf{S})$.

Then, by Lemma 2, there exists $\ddot{\eta}_{2} \in \Phi \ddot{\eta}_{1}$ such that

$\vartheta\left(\ddot{\eta}_{1}, \ddot{\eta}_{2}\right) \leq H_{\vartheta}\left(\Psi \ddot{\eta}_{0}, \Phi \ddot{\eta}_{1}\right)+q$.

Similarly, there exists $q$ in $(0,1)$ such that 
$H_{\vartheta}\left(\Phi \ddot{\eta}_{1}, \Psi \ddot{\eta}_{2}\right) \leq q \vartheta\left(\ddot{\eta}_{1}, \dddot{\eta}_{2}\right)+(1-q) \vartheta(\hat{\mathrm{R}}, S)$

and there exists $\ddot{\eta}_{3} \in \Psi \ddot{\eta}_{2}$ such that

$\vartheta\left(\ddot{\eta}_{2}, \ddot{\eta}_{3}\right) \leq H_{\vartheta}\left(\Phi \ddot{\eta}_{1}, \Psi \ddot{\eta}_{2}\right)+q^{2}$.

Repeating this way, one can create a sequence $\left\{\ddot{\eta}_{n}\right\}$ in Ŕ $\cup S$ such that $\ddot{\eta}_{2 n+1} \in \Psi \ddot{\eta}_{2 n}, \ddot{\eta}_{2 n+2} \in \Phi \ddot{\eta}_{2 n+1}$,

$\vartheta\left(\ddot{\eta}_{2 n+1}, \ddot{\eta}_{2 n+2}\right) \leq H_{\vartheta}\left(\Psi \ddot{\eta}_{2 n}, \Phi \ddot{\eta}_{2 n+1}\right)+q^{2 n+1}$

and

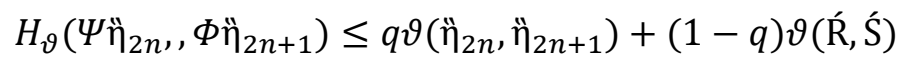

for all $n \in \mathbb{N}$. Hence, we have

$$
\begin{aligned}
\vartheta\left(\ddot{\eta}_{2 n+1}, \ddot{\eta}_{2 n+2}\right) & \leq H_{\vartheta}\left(\Psi \ddot{\eta}_{2 n}, \Phi \ddot{\eta}_{2 n+1}\right)+q^{2 n+1} \\
& \leq q \vartheta\left(\ddot{\eta}_{2 n}, \ddot{\eta}_{2 n+1}\right)+(1-q) \vartheta(\hat{\mathrm{R}}, \hat{S})+q^{2 n+1} \\
& \leq q\left(H_{\vartheta}\left(\Phi \ddot{\eta}_{2 n-1}, \Psi \ddot{\eta}_{2 n}\right)+q^{2 n}\right)+(1-q) \vartheta(\hat{\mathrm{R}}, \hat{\mathrm{S}})+q^{2 n+1} \\
& =q H_{\vartheta}\left(\Phi \ddot{\eta}_{2 n-1}, \Psi \ddot{\eta}_{2 n}\right)+(1-q) \vartheta(\mathrm{R}, \hat{S})+2 q^{2 n+1} \\
& \leq q^{2} \vartheta\left(\ddot{\eta}_{2 n-1}, \ddot{\eta}_{2 n}\right)+(1+q)(1-q) \vartheta(\hat{\mathrm{R}}, \hat{\mathrm{S}})+2 q^{2 n+1} \\
& \cdot \\
& \cdot \\
& \cdot \\
& \leq q^{2 n+1} \vartheta\left(\ddot{\eta}_{0}, \ddot{\eta}_{1}\right)+\left(1+q+\cdots+q^{2 n}\right)(1-q) \vartheta(\mathrm{R}, \hat{S})+(2 n+1) q^{2 n+1} \\
& \leq q^{2 n+1} \vartheta\left(\ddot{\eta}_{0}, \ddot{\eta}_{1}\right)+\frac{1-q^{2 n+1}}{1-q}(1-q) \vartheta(\mathrm{R}, \hat{S})+(2 n+1) q^{2 n+1} \\
& \leq q^{2 n+1} \vartheta\left(\ddot{\eta}_{0}, \ddot{\eta}_{1}\right)+\vartheta(\hat{\mathrm{R}}, \hat{S})+(2 n+1) q^{2 n+1} .
\end{aligned}
$$

Since the series $\sum_{n=0}^{\infty}(2 n+1) q^{2 n+1}$ is convergent for $q \in(0,1)$, we have $\lim _{n \rightarrow \infty}(2 n+1) q^{2 n+1}=0$. Taking limit $n \rightarrow \infty$ (2), we get

$\lim _{n \rightarrow \infty} \vartheta\left(\ddot{\eta}_{2 n+1}, \ddot{\eta}_{2 n+2}\right)=\vartheta(\hat{R}, \hat{S})$.

Similarly, we can show that

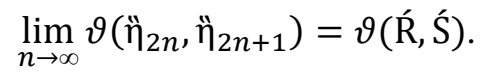

Hence, we have

$\vartheta\left(\ddot{\eta}_{n}, \ddot{\eta}_{n+1}\right) \rightarrow \vartheta(\hat{\mathrm{R}}, \hat{S})$ as $n \rightarrow \infty$.

Now, we give our main result.

Theorem 7. Let $\emptyset \neq R$, Ś be subsets of a pms $(\Omega, \vartheta)$. Assume that $\Psi: \hat{\mathrm{R}} \rightarrow C B^{\vartheta}\left(\mathcal{S}^{\prime}\right)$ and $\Phi: S \rightarrow C B^{\vartheta}(\hat{\mathrm{R}})$ are multivalued mappings satisfying the pair $(\Psi, \Phi)$ is a Hausdorff cyclic contraction mapping pair and $\ddot{\eta}_{0} \in$ Ŕ. Consider the sequence $\left\{\ddot{\eta}_{n}\right\}$ constructed as Proposition 1. Then, we get the following:

i) if the sequence $\left\{\varsigma_{2 n}\right\}$ has a subsequence $\left\{\varsigma_{2 n_{k}}\right\}$ in Ŕ such that 
$\lim _{k, l \rightarrow \infty} \vartheta\left(\ddot{\eta}_{2 n_{k}}, \ddot{\eta}_{2 n_{l}}\right)=\lim _{k \rightarrow \infty} \vartheta\left(\ddot{\eta}_{2 n_{k}}, \ddot{\eta}^{*}\right)=\vartheta\left(\dddot{\eta}^{*}, \ddot{\eta}^{*}\right)=0$

for some $\dddot{\eta}^{*} \in R$, then $\dddot{\eta}^{*}$ is a best proximity point of $\varphi$ in Ŕ.

ii) if the sequence $\left\{s_{2 n+1}\right\}$ has a subsequence $\left\{s_{2 n_{k}+1}\right\}$ in $S$ such that

$\lim _{k, l \rightarrow \infty} \vartheta\left(\dddot{\eta}_{2 n_{k}+1}, \dddot{\eta}_{2 n_{l}+1}\right)=\lim _{k \rightarrow \infty} \vartheta\left(\ddot{\eta}_{2 n_{k}+1}, \ddot{u}^{*}\right)=\vartheta\left(\ddot{v}^{*}, \ddot{u}^{*}\right)=0$

for some $\dddot{U}^{*} \in R$, then $\dddot{U}^{*}$ is a best proximity point of $\Phi$ in $S$.

Proof. Let $\left\{\ddot{\eta}_{n}\right\}$ be sequence constructed as Proposition 1. Assume that the condition (i) holds. Due to the condition (i), there is a subsequence $\left\{s_{2 n_{k}}\right\}$ of $\left\{s_{2 n}\right\}$ such that

$\lim _{k, l \rightarrow \infty} \vartheta\left(\ddot{\eta}_{2 n_{k}}, \ddot{\eta}_{2 n_{l}}\right)=\lim _{k \rightarrow \infty} \vartheta\left(\ddot{\eta}_{2 n_{k}}, \dddot{\eta}^{*}\right)=\vartheta\left(\dddot{\eta}^{*}, \dddot{\eta}^{*}\right)=0$

for some $\dddot{\eta ̆}^{*} \in$ Ŕ. From Proposition 1, we have

$\lim _{k \rightarrow \infty} \vartheta\left(\ddot{\eta}_{2 n_{k}}, \ddot{\eta}_{2 n_{k}+1}\right)=\vartheta(\hat{R}, S ́)$

Hence, we get

$$
\begin{aligned}
\vartheta(\hat{R}, \hat{S}) & \leq \vartheta\left(\dddot{\eta}^{*}, \ddot{\eta}_{2 n_{k}+1}\right) \\
& \leq \vartheta\left(\ddot{\eta}^{*}, \ddot{\eta}_{2 n_{k}}\right)+\vartheta\left(\ddot{\eta}_{2 n_{k}}, \ddot{\eta}_{2 n_{k}+1}\right)
\end{aligned}
$$

Letting limit $k \rightarrow \infty$, one have

$\vartheta\left(\dddot{\eta}^{*}, \dddot{\eta}_{2 n_{k}+1}\right) \rightarrow \vartheta(\hat{\mathrm{R}}, \hat{S})$ as $k \rightarrow \infty$

Therefore, we get

$$
\begin{aligned}
\vartheta(\mathrm{R}, S) & \leq \vartheta\left(\ddot{\eta}_{2 n_{k}+2}, \Psi \dddot{\eta}^{*}\right) \\
& \leq H_{\vartheta}\left(\Phi \ddot{\eta}_{2 n_{k}+1}, \Psi \dddot{\eta}^{*}\right) \\
& \leq q \vartheta\left(\ddot{\eta}_{2 n_{k}+1}, \dddot{\eta}^{*}\right)+(1-q) \vartheta(\mathrm{R}, \mathrm{S}) .
\end{aligned}
$$

Taking limit $k \rightarrow \infty$ in the last inequality, from (5), we have

$\vartheta\left(\dddot{\eta}^{*}, \Psi \dddot{\eta}^{*}\right)=\vartheta(\hat{R}, \dot{S})$.

Hence $\Psi$ has a best proximity point $\dddot{\eta}^{*}$ in Ŕ. Similarly, if the condition (ii) holds, then it can be shown that $\Phi$ has a best proximity point in Ś.

Recall that, a sequence $\left\{\ddot{\eta}_{n}\right\}$ in a pms $(\Omega, \vartheta)$ is bounded if there exist $a \in \Omega$ and $\mathrm{M}>0$ such that $\vartheta\left(\ddot{\eta}_{n}, a\right) \leq$ $\vartheta(a, a)+M$ for all $n \in \mathbb{N}$.

Proposition 2. Let $(\Omega, \vartheta)$ be a pms, $\varnothing \neq \hat{\mathrm{R}}, \hat{S} \subseteq \Omega$ and $\eta_{0} \in R$. Assume that $\Psi: \dot{\mathrm{R}} \rightarrow C B^{\vartheta}(\hat{S})$ and $\Phi: S \rightarrow$ $C B^{\vartheta}(\hat{\mathrm{R}})$ are multivalued mappings. If the pair $(\Psi, \Phi)$ is Hausdorff cyclic contraction mapping pair, then every sequence $\left\{\ddot{\eta}_{n}\right\}$ constructed as Proposition 1 is bounded.

Proof. Let $\left\{\ddot{\eta}_{n}\right\}$ be sequence constructed as Proposition 1. Then, since $\vartheta\left(\ddot{\eta}_{n}, \ddot{\eta}_{n+1}\right)$ converges to $\vartheta(\hat{R}, S$ S) by Proposition $1,\left\{\vartheta\left(\ddot{\eta}_{n}, \ddot{\eta}_{n+1}\right)\right\}$ is bounded sequence in $\mathbb{R}$. Since $\left\{\vartheta\left(\ddot{\eta}_{2 n-1}, \ddot{\eta}_{2 n}\right)\right\}$ is a subsequence of $\left\{\vartheta\left(\ddot{\eta}_{n}, \ddot{\eta}_{n+1}\right)\right\}$, we have 
$\lim _{n \rightarrow \infty} \vartheta\left(\dddot{\eta}_{2 n-1}, \dddot{\eta}_{2 n}\right)=\vartheta(\mathrm{R}, S)$

Hence, there exists $\mathrm{L}>0$ such that

$\vartheta\left(\ddot{\eta}_{2 n-1}, \ddot{\eta}_{2 n}\right) \leq L$

for all $n \geq 1$. Then, from Lemma 2 , there is $\dddot{\eta}_{1} \in \Psi \dddot{\eta ̆}_{0}$ satisfying

$$
\begin{aligned}
\vartheta\left(\ddot{\eta}_{2 n}, \ddot{\eta}_{1}\right) & \leq \vartheta\left(\ddot{\eta}_{2 n}, \Psi \ddot{\eta}_{0}\right)+(1-q) L \\
& \leq H_{\vartheta}\left(\Phi \ddot{\eta}_{2 n-1}, \Psi \ddot{\eta}_{0}\right)+(1-q) L \\
& \leq q \vartheta\left(\ddot{\eta}_{2 n-1}, \ddot{\eta}_{0}\right)+(1-q) \vartheta(\hat{\mathrm{R}}, \hat{\mathrm{S}})+(1-q) L \\
& \leq q \vartheta\left(\ddot{\eta}_{2 n-1}, \ddot{\eta}_{2 n}\right)+q \vartheta\left(\ddot{\eta}_{2 n}, \ddot{\eta}_{1}\right)+q \vartheta\left(\ddot{\eta}_{0}, \ddot{\eta}_{1}\right)+(1-q) \vartheta(\dot{\mathrm{R}}, \hat{S})+(1-q) L \\
& \leq q L+q \vartheta\left(\ddot{\eta}_{2 n}, \ddot{\eta}_{1}\right)+q \vartheta\left(\ddot{\eta}_{0}, \ddot{\eta}_{1}\right)+(1-q) \vartheta(\hat{\mathrm{R}}, \hat{S})+(1-q) L .
\end{aligned}
$$

Hence, we have

$$
(1-q) \vartheta\left(\ddot{\eta}_{2 n}, \ddot{\eta}_{1}\right) \leq L+q \vartheta\left(\ddot{\eta}_{0}, \ddot{\eta}_{1}\right)+(1-q) \vartheta(\hat{\mathrm{R}}, \dot{S})
$$

which implies

$$
\begin{aligned}
\vartheta\left(\ddot{\eta}_{2 n}, \ddot{\eta}_{1}\right) & \leq \frac{L}{(1-q)}+\frac{q \vartheta\left(\ddot{\eta}_{0}, \ddot{\eta}_{1}\right)}{(1-q)}+\vartheta(\hat{\mathrm{R}}, \hat{\mathrm{S}}) \\
& \leq \vartheta\left(\ddot{\eta}_{1}, \ddot{\eta}_{1}\right)+\frac{L}{(1-q)}+\frac{q \vartheta\left(\ddot{\eta}_{0}, \grave{\eta ̆}_{1}\right)}{(1-q)}+\vartheta(\hat{\mathrm{R}}, \hat{\mathrm{S}}) .
\end{aligned}
$$

Let

$M=\frac{L}{(1-q)}+\frac{q \vartheta\left(\ddot{\eta}_{0}, \ddot{\eta}_{1}\right)}{(1-q)}+\vartheta(\hat{\mathrm{R}}, \mathbf{S})$

Hence, $\left\{\ddot{\eta}_{2 n}\right\}$ is bounded. For each $n \in \mathbb{N}$, since

$$
\begin{aligned}
\vartheta\left(\ddot{\eta}_{2 n+1}, \ddot{\eta}_{1}\right) & \leq \vartheta\left(\ddot{\eta}_{2 n}, \ddot{\eta}_{2 n+1}\right)+\vartheta\left(\ddot{\eta}_{2 n}, \ddot{\eta}_{1}\right) \\
& \leq \vartheta\left(\ddot{\eta}_{1}, \ddot{\eta}_{1}\right)+L+M .
\end{aligned}
$$

Therefore, $\left\{\ddot{\eta}_{2 n+1}\right\}$ is bounded. Hence, $\left\{\ddot{\eta}_{n}\right\}$ is a bounded sequence in Ŕ $\cup$ Ś.

Now, we revise the definition of 0-boundedly compact on pms.

Definition 6. Let $(\Omega, \vartheta)$ be a pms and $\emptyset \neq \hat{\mathrm{R}} \subseteq \Omega$. Ŕ is called a 0 -boundedly compact if every bounded sequence $\left\{\ddot{\eta}_{n}\right\}$ has a subsequence $\left\{\ddot{\eta}_{n_{k}}\right\}$ such that

$\lim _{k, l \rightarrow \infty} \vartheta\left(\ddot{\eta}_{n_{k}}, \ddot{\eta}_{n_{l}}\right)=\lim _{k \rightarrow \infty} \vartheta\left(\ddot{\eta}_{n_{k}}, \ddot{\eta}^{*}\right)=\vartheta\left(\ddot{\eta}^{*}, \ddot{\eta}^{*}\right)=0$

for some $\dddot{\eta ̆}^{*} \in$ Ŕ.

Corollary 1. Let $(\Omega, \vartheta)$ be a pms and $\emptyset \neq \hat{\mathrm{R}}, \hat{S} \subseteq \Omega$. Assume that $\Psi: \hat{\mathrm{R}} \rightarrow C B^{\vartheta}\left(\hat{S}^{\prime}\right)$ and $\Phi: \hat{S}^{\mathrm{S}} \rightarrow C B^{\vartheta}(\hat{\mathrm{R}})$ are multivalued mappings satisfying the pair $(\Psi, \Phi)$ is a Hausdorff cyclic contraction mapping pair, then we get the following: 
i) if $R$ is a 0 -boundedly compact, then there is a point $\dddot{\eta}^{*}$ in $R$ satisfying $\vartheta\left(\dddot{\eta}^{*}, \Psi \dddot{\eta}^{*}\right)=\vartheta(R, S ́)$. Also, $\vartheta\left(\dddot{\eta}^{*}\right.$ , $\left.\ddot{\eta}^{*}\right)=0$.

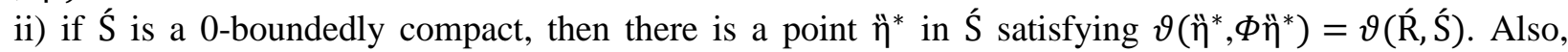
$\vartheta\left(\ddot{v}^{*}, \hat{v}^{*}\right)=0$.

Note that, although Ŕ and Ś are not 0-boundedly compact, a sequence satisfying (3) or (4) may be in Ŕ or $S$, respectively. The following example is important to show this fact.

Example 1. Let $\Omega=(\{0\} \cup[0,10]) \times(\{0\} \cup[0,10])$ and $\vartheta: \Omega \times \Omega \rightarrow[0, \infty)$ defined by

$$
\vartheta(\hat{\eta}, \hat{v})= \begin{cases}\frac{\hat{\eta}_{1}}{2}, & \hat{\eta}=\hat{v} \\ \hat{\eta}_{1}+\hat{v}_{1}+\left|\hat{\eta}_{2}-\hat{v}_{2}\right|, & \hat{\eta} \neq \hat{v}\end{cases}
$$

for all $\grave{\eta}=\left(\grave{\eta}_{1}, \grave{\eta}_{2}\right), \hat{v}=\left(\hat{v}_{1}, \hat{v}_{2}\right) \in \Omega$. It is clear that $(\Omega, \vartheta)$ is a pms. Let $\hat{\mathrm{R}}=(\{0\} \cup[2,4]) \times\{0\}$ and $\hat{S}=$ $[1,2) \times\{1\}$. Hence, we have $\vartheta(\hat{\mathrm{R}}, \hat{S})=2$. Now, we shall show that $\hat{\mathrm{R}}$ and $S$ are not 0 -boundedly compact. Indeed, let $\hat{\eta}_{n}=\left(2+\frac{1}{n}, 0\right)$ be a sequence in R. Then, $\left\{\hat{\eta}_{n}\right\}$ is a bounded sequence. But, there is no a subsequence $\left\{\ddot{\eta}_{n_{k}}\right\}$ of $\left\{\ddot{\eta}_{n}\right\}$ such that

$\lim _{k, l \rightarrow \infty} \vartheta\left(\grave{\eta}_{n_{k}}, \grave{\eta}_{n_{l}}\right)=\lim _{k \rightarrow \infty} \vartheta\left(\grave{\eta}_{n_{k}}, \ddot{\eta}^{*}\right)=\vartheta\left(\ddot{\eta}^{*}, \hat{\eta}^{*}\right)=0$

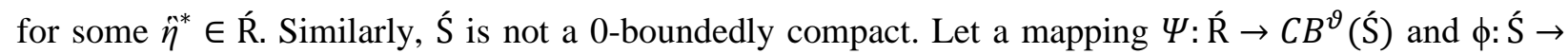
$C B^{\vartheta}(\mathrm{R})$ defined by

$\Psi \hat{\eta}=\left[\frac{\hat{\eta}_{1}+8}{8}, \frac{\hat{\eta}_{1}+6}{6}\right] \times\{1\}$

for all $\grave{\eta}=\left(\grave{\eta}_{1}, 0\right) \in R$ and

$\Phi \ddot{i}=\{(0,0)\}$

for all $i=\left(i_{1}, 1\right) \in$ S. Now, we shall show that the pair $(\Psi, \Phi)$ is a Hausdorff cyclic contraction mapping pair for $q=\frac{1}{6}$. Indeed, for all $\hat{\eta}=\left(\hat{\eta}_{1}, 0\right) \in \mathrm{R}$ and $\hat{v}=\left(\hat{v}_{1}, 1\right) \in S$, we have

$$
\begin{aligned}
H_{\vartheta}(\Psi \ddot{\eta}, \Phi \hat{v}) & =\max \left\{\sup _{a \in \Psi \dot{\eta}} \vartheta(a, \Phi \hat{v}), \sup _{b \in \Phi i \dot{v}} \vartheta(\Psi \ddot{\eta}, b)\right\} \\
& =\frac{\hat{\eta}_{1}+6}{6}+0+1 \\
& \leq \frac{1}{6}\left(\hat{\eta}_{1}+\hat{v}_{1}+1\right)+\left(1-\frac{1}{6}\right) 2 \\
& =q \vartheta(\hat{\eta}, \hat{v})+(1-q) \vartheta(\mathrm{R}, \hat{S}) .
\end{aligned}
$$

Hence, the pair $(\Psi, \Phi)$ is a Hausdorff cyclic contraction mapping pair. Moreover, we choose

$\grave{\eta}_{0}=(a, 0), \quad \grave{\eta}_{1}=(b, 1) \in \Psi \grave{\eta}_{0}=\left[\frac{a+8}{8}, \frac{a+6}{6}\right] \times\{1\}, \quad \ddot{\eta}_{2}=(0,0) \in \Phi \grave{\eta}_{1}, \ldots, \grave{\eta}_{2 n}=(0,0), \ldots$

Hence, $\left\{\ddot{\eta}_{2 n}\right\}$ has a subsequence, $\left\{\ddot{\eta}_{2 n_{k}}\right\}$ such that

$\lim _{k, l \rightarrow \infty} \vartheta\left(\grave{\eta}_{2 n_{k}}, \grave{\eta}_{2 n_{l}}\right)=\lim _{k \rightarrow \infty} \vartheta\left(\grave{\eta}_{2 n_{k}},(0,0)\right)=\vartheta((0,0),(0,0))=0$ 
Therefore, all the conditios of Theorem 7 hold, and therefore $\Psi$ has a best proximity point $\eta^{*}=(0,0)$ in Ŕ.

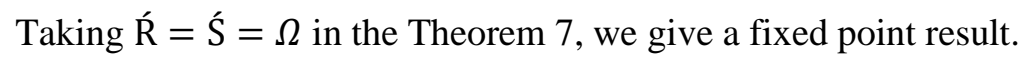

Corollary 2. Let $\Psi, \Phi: \Omega \rightarrow C B^{\vartheta}(\Omega)$ be multivalued mappings on a pms $(\Omega, \vartheta)$ and satisfying the pair $(\Psi, \Phi)$ is a Hausdorff cyclic contraction mapping pair. Let $\ddot{\eta}_{0} \in \Omega$ and define a sequence $\left\{\ddot{\eta}_{n}\right\}$ by $\ddot{\eta}_{n+1}=$ $\ddot{\eta}_{n}$ for all $n \geq 1$. If $\left\{\hat{\eta}_{2 n}\right\}$ has a subsequence in $\Omega$ such that

$\lim _{k, l \rightarrow \infty} \vartheta\left(\grave{\eta}_{2 n_{k}}, \grave{\eta}_{2 n_{l}}\right)=\lim _{k \rightarrow \infty} \vartheta\left(\grave{\eta}_{2 n_{k}}, \ddot{\eta}^{*}\right)=\vartheta\left(\ddot{\eta}^{*}, \ddot{\eta}^{*}\right)=0$

for all $\hat{\eta}, \hat{v} \in \Omega$, then $\hat{\eta}^{*}$ is a fixed point of $\Psi$ in $\Omega$.

If we take $\dot{R}=S=\Omega$ and $\vartheta(\hat{R}, S)=0$ in Theorem 7 , then we can show that every sequence $\left\{\ddot{\eta}_{n}\right\}$ constructed as Proposition 1 in $\Omega$ is a 0 -Cauchy sequence. Hence, by accepting the completeness of $\Omega$, sequence $\left\{\hat{\eta}_{n}\right\}$ constructed as Proposition 1 has a convergent subsequence. Therefore, we present the following fixed point result.

Corollary 3. Let $\Psi, \Phi: \Omega \rightarrow C B^{\vartheta}(\Omega)$ be a multivalued mappings on 0 -complete pms $(\Omega, \vartheta)$. If there exists $q$ in $(0,1)$ such that

$H_{\vartheta}(\Psi \ddot{\eta}, \Phi \ddot{v}) \leq q \vartheta(\ddot{\eta}, \ddot{v})$

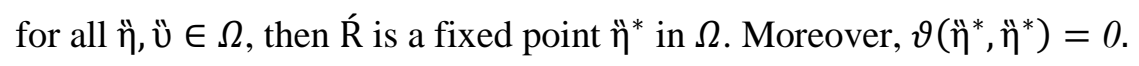

\section{CONFLICTS OF INTEREST}

The author declares that there is no conflict of interest.

\section{REFERENCES}

[1] Banach, S., "Sur les opérations dans les ensembles abstraits et leur applications aux équations intégrales", Fundamenta Mathematicae, 3: 133-181, (1922).

[2] Kadelburg, Z., Radenovic, S., "Fixed point and tripled fixed point theorems under Pata-Type conditions in ordered metric spaces", International Journal of Analysis and Applications, 6: 113$122,(2014)$.

[3] Reich, S., "Fixed points of contractive functions", Bollettino Della Unione Matematica Italiana, 5: 26-42, (1972).

[4] Cevik, C., Altun, I., Sahin, H., Ozeken, C.C., "Some fixed point theorems for contractive mapping in ordered vector metric spaces", Journal of Nonlinear Sciences and Applications, 10(4): 14241432, (2017).

[5] Altun, I. ,Sahin, H., Turkoglu, D., "Fixed point results for multivalued mappings of Feng-Liu type on $M$-metric spaces", Journal of Nonlinear Functional Analysis, 2018: 1-8, (2018).

[6] Altun, I., Sahin, H., Turkoglu, D., "Caristi-Type fixed point theorems and some generalizations on $M$-metric space", Bulletin of the Malaysian Mathematical Sciences Society, 43: 2647-2657, (2020).

[7] Nadler, S. B., "Multivalued contraction mappings", Pacific Journal of Mathematics, 30(2): 475$488,(1969)$. 
[8] Kirk, W. A., Srinivasan, P. S., Veeramani, P., "Fixed points for mappings satisfying cyclical contractive conditions", Fixed Point Theory, 4: 79-89, (2003).

[9] Karapinar, E., Samet, B., "Generalized $\alpha-\psi$ contractive type mappings and related fixed point theorems with applications", In Abstract and Applied Analysis, Hindawi Limited, (2012).

[10] Petrusel, G., "Cyclic representations and periodic points", Studia University Babes-Bolyai, Math, 50: 107-112, (2005).

[11] Basha, S. S., Veeramani, P., "Best approximations and best proximity pairs", Acta Scientiarum Mathematicarum, 63: 289-300, (1977).

[12] Abkar, A., Gabeleh, M., "The existence of best proximity points for multivalued nonselfmappings", Revista de la Real Academia de Ciencias Exactas, Fisicas y Naturales.Serie A. Matematicas, 107(2): 319-325, (2013).

[13] Altun, I., Aslantas, M., Sahin, H., "Best proximity point results for p-proximal contractions", Acta Mathematica Hungarica, 162: 393-402, (2020).

[14] Basha, S. S., "Extensions of Banach's contraction principle", Numerical Functional Analysis and Optimization, 31 (5): 569-576, (2010).

[15] Sahin, H., Aslantas, M., Altun, I., "Feng-Liu type approach to best proximity point results for multivalued mappings", Journal of Fixed Point Theory and Applications, 22, (2020).

[16] Aslantas, M., "Best proximity point theorems for proximal b-cyclic contractions on b-metric spaces", Communications Faculty of Sciences University of Ankara Series A1 Mathematics and Statistics, 70(1): 483-496, (2021).

[17] Iş1k, H., Aydi, H., "Best proximity problems for new types of Z-proximal contractions with an application", Communications Faculty of Sciences University of Ankara Series A1 Mathematics and Statistics, 69(2): 1405-1417, (2020).

[18] Işık, H., Aydi, H., Mlaiki, N., Radenović, S., "Best proximitiy point results for Geraghty type Zproximal contractions with an application", Axioms, 8(3): 81, (2019).

[19] Iş1k, H., Sezen, M. S., Vetro, C., " $\Psi$-Best proximity point theorems and applications to variational inequality problems", Journal of Fixed Point Theory and Applications, 19(4): 3177-3189, (2017).

[20] Sahin, H., "Best proximity point theory on vector metric spaces", Communications Faculty of Sciences University of Ankara Series A1 Mathematics and Statistics, 70(1): 130-142, (2021).

[21] Eldred, A. A., Veeramani, P., "Existence and convergence of best proximity points", Journal of Mathematical Analysis and Applications, 323: 1001-1006, (2006).

[22] Matthews, S. G., "Partial metric topology", Annals of the New York Academy of Sciences-Paper Edition, 728: 183-197, (1994).

[23] Abbas, M., Nazir, T., "Fixed point of generalized weakly contractive mappings in ordered partial metric spaces", Fixed Point Theory Applications, 2012(1): 19, (2012).

[24] Altun, I., Sola, F., Simsek, H., "Generalized contractions on partial metric spaces", Topology and its Applications 157(18): 2778-2785, (2010). 
[25] Altun, I., Simsek, H., "Some fixed point theorems on dualistic partial metric spaces", Journal of Advanced Mathematical Studies, 1(1-2): 1-9, (2008).

[26] Romaguera, S., "A Kirk type characterization of completeness for partial metric spaces", Fixed Point Theory and Applications, 2009(1): 493298, (2010).

[27] Abdeljawad, T., Alzabut, J., Mukheimer, A., Zaidan, Y., "Best proximity points for cyclical contraction mappings with 0-boundedly compact decompositions", Journal of Computational analysis and Applications, 15: 678-685, (2013).

[28] Aydi, H., Abbas, M.,Vetro, C., "Partial Hausdorff metric and Nadler's fixed point theorem on partial metric spaces", Topology and its Applications, 159(14): 3234-3242, (2012). 